\title{
Review
}

\section{Research progress in the targeted monitoring of surgical site infections}

Pingdong Jia

Department of Respiratory Medicine, Jinan Central Hospital, Jinan, China

\author{
Keywords \\ Surgical site infection, Risk factors, Prevention \\ measures \\ Correspondence \\ Pingdong Jia, \\ E-mail: jiapdfy@163.com \\ DOI: 10.1515/ii-2017-0137
}

\begin{abstract}
Surgical treatment can decrease the pain burden of patients, as well as improve their quality of life and social satisfaction. However, postoperative infection and other complications may harm patients and may even cause surgical failure and death. This review describes the definition, classification, and status of surgical site infection (SSI). In addition, this review provides an analysis of the relevant risk factors that result in SSI, and proposes the corresponding preventative and control measures for each different risk factor.
\end{abstract}

Surgical patients usually exhibit postoperative weakness and low resistance. When accompanied by invasive surgery, the extensive use of antibacterial drugs causes dysbacteriosis and the propagation of drug-resistant strains. The above factors all increase the probability of hospital infection. Cases of postoperative surgical site infection (SSI) account for $15 \%$ of reported cases of hospital infection. The incidence of SSI ranks third among those of all types of hospital infection. SSI increases the length of hospital stay, patient mortality, and hospitalization expenses, thus exerting considerable economic burden on individuals and society. More than $40 \%$ of SSI cases will benefit from SSI monitoring. ${ }^{[1]}$ Therefore, studying the incidence and risk factors of SSI are crucial for the prevention and control of hospital infection and increase investment in the control of SSI by raising awareness among the administrative and management departments of hospitals. Controlling SSI can decrease hospital infection rates, relieve the pain burden of patients, as well as improve the health and decrease the medical expenses of patients.

\section{Definition and classification of SSI}

SSI is defined as infection that develops in surgical incision and organs. According to the 2001 Hospital Infection Diagnosis issued by the National Health and Family Planning Commission of PRC, SSI is divided into 3 categories: infection of superficial incisions, deep incisions, or organ/lacunae. Superficial incision infection is limited to infections of the incisions of the skin and subcutaneous tissue and occurs within 30 days of surgery. Deep incision infection refers to infection that occurs 30 days within nonimplant surgery or within 1 year of implant surgery, such as the installation of cardiac valve prostheses, artificial blood vessels, mechanical hearts, and artificial joints. Deep incision infection occurs in deep soft tissue, such as deep fascia and muscle. Organ/lacunar infection refers to the infection of organs or lacunae, except for the skin, subcutaneous tissue, deep fascia, or muscle, within 30 days of non-implant surgery and within 1 year of implant surgery.

\section{Current status of SSI}


As reported by the World Health Organization, the global incidence of SSI is approximately $0.50 \%-15.00 \%$ and is not equally distributed. Wu Anhua et al. pointed out that in China, the incidence of SSI ranks third among those of other hospital infections. On average, each incidence of SSI extends hospital stay by nearly 12 days and increases treatment costs by $12872 \mathrm{RMB}^{[2]}$. Therefore, the prevention and control of SSI has become an important part of hospital infection management.

\section{Risk factors of SSI}

The study of SSI risk factors is crucial for the prevention and control of SSI. The infection of surgical incisions depends on numerous factors. In 2002, the Center for Disease Control identified basic situation and operation factors as SSI risk factors ${ }^{[3]}$. Numerous clinical studies and observations have established that the incidence of postoperative infection is related with patient factors, surgery factors, environmental and sanitary factors, hand hygiene, and the unreasonable application of antibacterial drugs ${ }^{[4]}$.

\section{Patient factor}

The inherent factors of the patient can increase the probability of SSI. Physiological factors, such as age and obesity, and underlying diseases, such as diabetes, malignant tumors, and chronic obstructive pulmonary disease (COPD), are some obvious factors of SSI.

\section{Age}

A large number of literatures show that SSI easily occurs among infants and elderly patients ${ }^{[6]}$. The young age and immature immune organs of infants facilitates SSI. In the elderly, the functional decline of immune organs facilitates SSI.

\section{Obesity}

Obese patients have high body fat, poor blood circulation, and low healing rate. The intra-operative incision on both sides of tissue is under high tension. Mechanical actions like extrusion and clamping can injure fat tissue. In addition, the dead space that results from poor stitching increases the probability of infection. Some literatures report that the probability and severity of postoperative infection, a highrisk factor of SSI, among obese patients is higher than that among normal patients ${ }^{[7]}$.

\section{Underlying diseases}

1) Diabetes: Hyperglycemia is an independent risk factor of SSI ${ }^{[8]}$. Hyperglycemia not only restrains the phagocytic function of leukocytes and facilitates infection but also causes blood stream obstruction and the tissue ischemia hypoxia of peripheral vessels. The high blood sugar levels of diabetes patients provide a natural culture medium for bacterial propagation, thus facilitating infection. In addition, the difficulty of postoperative healing among diabetes patients increases the chances of infection. Studies have shown that once the blood sugar of a liver transplant patient exceed $11.2 \mathrm{mmol} / \mathrm{L}$, the incidence rate of SSI increases $(\mathrm{OR}=2.25,95 \% \mathrm{CI} 1.26-4.03, P=0.006)$ [9]. The incidence of SSI among diabetes patients after the surgical installation of artificial joints is approximately thrice that among non-diabetes patients ${ }^{[10]}$. 2) Malignant tumors: The incidence of malignant tumors has gradually increased in recent years. Therefore, malignant tumors have become a clinically common and frequently occurring disease. Tumors, particularly malignant tumors, are a wasting disease that degrades the immune system of patients. In addition, the toxic products of tumors also can cause metabolic disorders. Radiotherapy and chemotherapy will decrease leukocyte counts and increase the chances of infection. The incidence rate of hospital infection after tumor surgery is $39.76 \%{ }^{[11]}$. 3) COPD: Elderly patients have low immunity. Thus, if their disease is combined with infective lesions like COPD, the infection decreases the efficacy of surgery. COPD can increase the comparative risk of postoperative pulmonary infection by $2.70 \%-4.70 \%{ }^{[12]}$.

\section{Environmental and sanitary factors}

The operating room is the key area of hospital infection. The air quality of the operating room directly influences wound healing. The high number of operations, frequent consecutive operations, and the reception and delivery of patients all cause air pollution and result in postoperative 
infection ${ }^{[13]}$. The case history pages and CT photography can introduce contamination; incomplete disinfection, broken aseptic pack, or expired aseptic materials can all cause hospital infection.

\section{Hand hygiene}

Direct or indirect infection results from hand contact, which is the most common transmission pathway of hospital infection. Insufficient hand hygiene facilities and the poor implementation of hand hygiene methods can cause the transmission of pathogenic bacteria. Approximately $30 \%$ of hospital infections are transmitted via hand contact among medical staff ${ }^{[14]}$.

\section{Relevant surgical factors}

\section{Preoperative skin preparation}

Improper preoperative skin preparation increases the probability of infection. Research has shown that the traditional shaving method easily injures the skin, thus destroying skin integrity and increasing the probability of infection ${ }^{[15]}$.

\section{Surgical site}

Some literatures show that in surgeries with the same risk level, patients who underwent colon, rectum, or gallbladder resection have the highest incidence of SSI. SSI incidence is particularly high among patients who underwent gastrointestinal surgery and accounts for more than $90 \%$ of all cases of incision infection ${ }^{[16]}$. Pathogenic bacteria colonize the colon and rectum, and the content of the digestive tract easily contaminates the surgical site.

Anesthesia method Some studies have reported that the postoperative lower respiratory infection of patients under general anesthesia is higher than that in patients under other anesthesia methods. Intubation for general anesthesia connects the respiratory tract with the external environment and makes the nose and mouth pharynx lose their defensive role ${ }^{[17]}$. In addition, general anesthesia can cause lesions in the tracheal epithelia, thus increasing the chances of bacterial infection.

\section{Emergency surgery}

Patients that require emergency surgery usually are in a critical condition. Cases that require emergency surgery include stroke, vehicular accidents, and viscera perforation. Emergency surgery patients are usually in poor condition with low immunity and may even present infection symptoms. In the meantime, the insufficient preparation of the operating room, the incomplete disinfection of indoor air, the insufficient hand hygiene of the medical staff under emergency circumstances, and the irregular operation of aseptic techniques all will increase the probability of infection. Therefore, the incidence of infection after emergency surgery is often higher than that after elective surgery ${ }^{[18]}$.

\section{Surgical time}

1) Duration of preoperative hospital stay: Extended preoperative hospitalization is an independent risk factor for SSI. Extended hospitalization increases the probability of colonization by drug-resistant strains, which easily cause hospital infection ${ }^{[19]}$. 2) Surgical duration: Extended surgery increases the chances that the incision comes with contact with microbes from the external environment. In addition, extended traction causes edema damage to tissue at the surgical site, thus influencing the healing effect and increasing the probability of infection. Some reports have verified that SSI is proportional to the duration of surgery. Anaya et al. ${ }^{[20]}$ discovered that the duration of surgery $\geq$ 2 hours is an independent risk factor for postoperative infection.

\section{Intra-operative warming}

Wogan etc. ${ }^{[21]}$ reported that during surgery, patients with hypothermia are thrice more likely to obtain SSI. The hospital stay of these patients will be prolonged by approximately $20 \%$. The low temperature in the operating room, the inhibiting effect of the anesthetic drug on the heat-regulating center, intra-operative transfusion, the exposure of the body surface and cavity, and normal saline washing will all cause intra-operative hypothermia, which decreases body immunity and increases the probability of infection.

\section{Postoperative intervention}

Physicians and interns with low qualifications and poor, 
unstandardized aseptic operation are usually responsible for postoperative dressing. They usually cannot accurately judge the status of wound healing, thus easily causing SSI [22].

\section{Preventive application of antibacterial drug}

Applying antibacterial drugs $30 \mathrm{~min}-2 \mathrm{~h}$ before surgery can effectively prevent the incidence of SSI. However, the extended postoperative use of antibacterial drugs not only fails to decrease the incidence of SSI but instead increases the probability of infection. The injudicious use of antibacterial drugs without indications, the inaccurate timing and supplementation during operation, the longtime use of antibacterial drugs after operation, and combination drug therapy without indications will all increase the probability of hospital infection ${ }^{[23]}$.

\section{Prevention and control measures of SSI}

To summarize, SSI is caused by multiple factors. To effectively prevent the incidence of SSI, training on knowledge about hospital infection should be strengthened. In addition, the understanding of prevention and control measures of SSI by the medical staff should be enhanced and targeted measures should be adopted.

\section{Improve patient factors}

Actively treat the basic diseases and primary lesions of patients. Provide additional nutrition to patients to strengthen their immunity and maintain their sound conditions before operation.

\section{Environmental and sanitary factors}

Strictly allocate functional areas, standardize the management of disposable items, regularly maintain the air disinfection machine, and strengthen the ventilation of the operating room. Clean and disinfect the medical facilities, contaminated items, surfaces of items, and the floor.

\section{Standardize hand hygiene:}

Set a standard for hand hygiene. Strictly implement hand disinfection in accordance with the hand disinfection requirements of the surgery department. Regularly or irregularly implement the hand surface bacterial culture to ensure that hand hygiene has effectively decreased the transmission of infection via hand contact. To decrease the incidence of hospital infection and to prevent cross infection through the medical staff, strengthen the knowledge training on hand hygiene and enhance the compliance of medical staff to hand hygiene.

\section{Relevant factors of surgical operation}

\section{Reasonable preoperative skin preparation}

Studies have shown that preoperative skin preparation should be as brief as possible. The traditional shaving method for skin preparation will injure the skin and increase the probability of infection. A depilatory has a lower probability for infection than shaving but may cause adverse reactions. Therefore, the shearing method, which neither injures the skin nor causes skin allergy, is the best skin preparation method and should be used as much as possible ${ }^{[24]}$. Preoperative skin preparation also includes skin cleaning. If $2 \%$ gluconate chlorhexidine is applied during the preoperative shower, it can achieve 7 days of antibacterial effect. To achieve sterilization effect, patients for elective surgery should use $2 \%$ gluconate chlorhexidine for bathing on the night before the operation and on the morning of operation day.

\section{Improve surgical techniques}

Surgeons should strengthen their training, standardize their techniques, correctly evaluate the condition of the patients, and use reasonable anesthesia. Surgery should be conducted in accordance with aseptic techniques to decrease unnecessary contamination. Sufficient operative exposure can decrease tissue damage. Close cooperation with the instrument nurses can decrease the duration of surgery. Surgeons should avoid residual cavities when stitching and allow for adequate drainage after surgery to decrease the incidence of postoperative infection. The air conditioner should be turned on during surgery. Cover the body of the patient with a blanket and provide a hot-water bottle at the venous transfusion position to maintain he intra-operative body temperature of the patients, reduce the infection probability caused by hypothermia, and to 
shorten hospital stay and bed rest time.

\section{Strengthen postoperative intervention}

Given that lowly qualified physicians and interns are responsible for changing postoperative dressing, the training of newcomers and interns should be improved, aseptic techniques should be standardized, and regular spot check and assessments should be conducted.

\section{Management of antibacterial drug application}

Strictly implement antibacterials in accordance with the requirements in Guidelines for Clinical Use of Antibacterial and Notice of Related Issues about Clinical Application of Antibacterial Drug Management announced by General Office of the Ministry of Health. Class I surgery does not require the prophylactic use of antibacterial drugs. Class II surgery requires $24 \mathrm{~h}$ of the prophylactic use of antibacterial drugs, which can be extended to $48 \mathrm{~h}$ if necessary. The prophylactic use of drugs in Class III surgery can be appropriately extended in accordance with the situation. As the perioperative prophylactic, antibacterial drugs should be selected based on the type of surgery, surgical site, and antibacterial spectrum. Medication should be provided at the appropriate moment (30-120 min before surgery) and stopped as soon as possible after surgery to ensure the appropriate and rational application of antibacterials. In the meantime, clinical doctors should inspect and select the right antibacterial drug in accordance with the results of drug sensitivity tests to decrease bacterial drug resistance and treatment failure from the injudicious use of drugs.

\section{Declarations}

\section{Acknowledgements}

No.

\section{Competing interests}

The author declares that he has no competing interest.

\section{Authors' contributions}

PD Jia made the literature analysis and wrote, discussed and revised the manuscript of this review.

\section{References}

1 Ren N, Feng L, Wen X, et al. Practical Hospital Infection Monitoring Methodology, Changsha: Hunan Science and Technology Press, 2012.

2 Huang W, Chen $\mathrm{H}, \mathrm{Chu} \mathrm{L}$, et al. The loss of economic evaluation for surgical Site infection Clinical and experimental Medical Journal, 2010,9(9):655-657.

3 Malone DL, Genuit T, Tracy JK, et al. Surgical site infections: reanalysis of risk factors. J Surg Res, 2002, 103(1): 89-95.

4 Liu Y. Progress on the prevention and controlling of the surgical site infection. The Chinese Journal of Clinical Physicians: E-version, 2012, 6(8):1978-1981.

5 Fiorio M, Marvaso A, Vigano F, et al. Incidence of surgical site infections in general surgery in Italy. Infection, 2006, 34(6):310-314.

6 Kaye KS, Sloane R, Sexton DJ, et al. Risk factors for surgical site infections in older people. J Am Geriatr Soc, 2006, 54(3):391-396.

7 Dindo D, Muller MK, Weber M, et al. Obesity in general elective surgery. Lancet, 2003, 361(9374): 2032-2035.

8 Qadan M, Akca O, Mahid SS, et al. Perioperative supple-mental oxygen therapy and surgical site infection:a meta-analysis of randomizde controlled trials. Arch Surg, 2009, 144(4):359-366.

9 Park C, Hsu C, Neelakanta G, et al. Severe intraoperative hyperglycemia is independently associated with surgical site infection after liver transplantation. Transdantation, 2009, 87(7): 1031-1036.

10 Malinzak RA, Ritter MA, Berend ME, et al. Morbidly obese, diabetic, younger, and unilateral joint arthroplasty patients have elevated total joint arthroplasty rates. J Arthroplasty, 2009, 24(6Suppl):84-88.

11 Li J, Xiao X, Hua Y, et al., Analysis and treatment measures on the incision infection of the gastrointestinal surgery of the elderly patients. Chinese Journal of Nosocomiology, 2012, 22(10): 2078-2079.

12 Zhao $\mathrm{H}$, Wang $\mathrm{H}, \mathrm{Yu}$, et al. Risk factor analysis on the intrapulmonary infection of the postoperative coma patients with cerebral hemorrhage after tracheotomy. Chinese Journal of Nosocomiology, 2012,22(8):1580-1582.

13 Jiang M. Reduce the infection probability of surgical incision by strengthening the control measures to the operating room infection. Chinese Journal of Nosocomiology, 2013, 23(4): 890-891.

14 Sun L, Liu C, Liu S, et al. Monitoring analysis on the hand hygiene effect of the medical staffs. Chinese Journal of Nosocomiology, 2008, 18(7): 958-959.

15 Murta EF, Silva CS, Junior OR. Frequency of glove perforation and the protective effect of double gloves in gynecological surgery. Arch Gynecol Obstet, 2003, 268(2):82-84.

16 Mishriki SF, Law DJ, Jeffery PJ. Factor affecting the incidence of postoperative wound infection. J Hosp Infect, 1990, 16(3):223-230.

17 Chen $S$, Chen Q. Clinical analysis and countermeasures to the low 
respiratory tract infection after the surgical anesthesia. Chinese Journal of Nosocomiology, 2008, 18(7): 958-959.

18 Sheng F, Clinical Feature research and prevention measures of the incision infection of orthopedic surgery. Chinese Journal of Nosocomiology, 2012, 22(20): 4513-4514.

19 Zhang X. Risk factor analysis and countermeasures on the incision site infection of the general surgery patients. Chinese Journal of Nosocomiology, 2013, 23(5): 1041-1043.

20 Anaya DA, Cormier JN, Xing Y, et al. Development and validation of a novel stratification tool for identifying cancer patients at increased risk of surgical site infection, 2012, 255(1): 134-139.

21 Wogan GN, Dedon PC, Tannenbaum SR, et al. Infection, inflammation and colon carcinogenesis. Oncotarget, 2012, 3(8): $737-738$

22 Fan $\mathrm{M}, \mathrm{Ye} \mathrm{W}, \mathrm{Xu}$ X, et al. Influence of comprehensive warming measures on the postoperative infection incidence of the intestinal cancer patients. Chinese Journal of Nosocomiology, 2013, 23(50): 1054-1063.

23 Sun J. Targeted monitoring research on the surgical incision infection. Anhui Medical Journal, 2009, 30(4): 464-465.

24 Ko W, Lazenby WD, Zelano JA, et al. Effects of shaving methods and intraoperative irrigation on suppurative mediastinitis after bypass operations. Ann Thorac Surg, 1992, 53(2): 301-305. 